University of Wollongong

Research Online

Australian Institute for Innovative Materials -

Papers

Australian Institute for Innovative Materials

$1-1-2018$

Super-compatible functional boron nitride nanosheets/polymer films with excellent mechanical properties and ultra-high thermal conductivity for thermal management

\author{
Jiemin Wang \\ Deakin University \\ Yuanpeng Wu \\ Deakin University \\ Ye Xue \\ Rowan University \\ Dan Liu \\ Deakin University \\ Xuebin Wang \\ Nanjing University
}

See next page for additional authors

Follow this and additional works at: https://ro.uow.edu.au/aiimpapers

Part of the Engineering Commons, and the Physical Sciences and Mathematics Commons

Research Online is the open access institutional repository for the University of Wollongong. For further information contact the UOW Library: research-pubs@uow.edu.au 


\title{
Super-compatible functional boron nitride nanosheets/polymer films with excellent mechanical properties and ultra-high thermal conductivity for thermal management
}

\author{
Abstract \\ High heat-dissipation polymers are currently in great demand especially with the rapid development of \\ electronic devices. However, traditional polymer composites usually suffer from both low thermal \\ conductivity due to poor dispersibility and low concentration of fillers in the polymer matrix. To address \\ this issue, it is necessary to improve the compatibility between the thermal conductive fillers and polymer \\ matrix. Here, we designed a highly water-soluble functionalized boron nitride (FBN) nanosheet. Unlike \\ most functional $B N$ nanosheets that are only dispersible in water with polymer matrix at low \\ concentrations, our FBN nanosheets can be mutually dispersed with aqueous polymers such as polyvinyl \\ alcohol (PVA) in arbitrary weight ratios. The super compatibility between FBN and PVA is further \\ interpreted by the Pickering emulsion formed from water and n-hexane. Moreover, after facile vacuum \\ filtration, the robust FBN/polymer freestanding films with layer-by-layer laminate nanostructures are well \\ fabricated. The nanocomposite films exhibit superior in-plane thermal conductivity $(120 \mathrm{~W} \mathrm{~m}-1 \mathrm{~K}-1$ for \\ $90 \mathrm{wt} \%$ FBN loading in FBN/PVA film), which is nearly 100 times larger than that of the pristine PVA film. \\ The FBN/polymer films provide good fire-retardant ability, thus effectively retarding flammability. In \\ addition, the nanocomposite film with high concentration of FBN up to $70 \mathrm{wt} \%$ still possessed excellent \\ flexibility and toughness even after being rolled and folded 100 times. Interestingly, the rolled film hollow \\ cylinder supported 25:000 times its own weight without cracking, highlighting the extra strong interaction \\ between the FBN and PVA. These properties make the nanosheet an excellent candidate for thermal \\ management in electronics. \\ Disciplines \\ Engineering | Physical Sciences and Mathematics

\section{Publication Details} \\ Wang, J., Wu, Y., Xue, Y., Liu, D., Wang, X., Hu, X., Bando, Y. \& Lei, W. (2018). Super-compatible functional \\ boron nitride nanosheets/polymer films with excellent mechanical properties and ultra-high thermal \\ conductivity for thermal management. Journal of Materials Chemistry C, 6 (6), 1363-1369.

\section{Authors} \\ Jiemin Wang, Yuanpeng Wu, Ye Xue, Dan Liu, Xuebin Wang, Xiao Hu, Yoshio Bando, and Weiwei Lei
}

This journal article is available at Research Online: https://ro.uow.edu.au/aiimpapers/2942 
Received 00th January 20xx Accepted 00th January 20xx DOI: $10.1039 / \times 0 \times x 00000 x$

www.rsc.org/

\title{
Super compatible functional BN nanosheets/polymer films with excellent mechanical property and ultra-high thermal conductivity for thermal management
}

\author{
Jiemin Wang, ${ }^{\text {ta }}$ Yuanpeng Wu, ${ }^{\dagger a, b}$ Ye Xue, ${ }^{c}$ Dan Liu, ${ }^{* a}$ Xuebin Wang, d,e Xiao Hu, ${ }^{c}$ Yoshio Bando, ${ }^{\text {d,f }}$ \\ Weiwei Lei ${ }^{*}$
}

\begin{abstract}
Recently, high heat dissipation polymers are in huge demand especially with the rapid development of electron devices. However, traditional polymer composites usually suffer from both low thermal conductivity due to poor dispersibility and low concentration of fillers in the polymer matrix. To address this issue, it is principle to improve the compatibility between thermal conductive fillers and polymer matrix. Here we design a highly water-soluble functionalized boron nitride (FBN) nanosheets. Unlike most functional BN nanosheets which are only dispersible in water with polymer matrix at low concentrations, our FBN nanosheets could be mutually dispersed with aqueous polymers such as polyvinyl alcohol (PVA) in arbitrary weight ratios. The super compatibility of FBN and PVA is further interpreted by Pickering emulsion formed from water and $n$-hexane. Moreover, after facile vacuum filtration, the robust FBN/polymer freestanding films with layer by layer laminate nanostructures are well fabricated. The nanocomposite films exhibit superior in-plane thermal conductivity (120 $\mathrm{W} \mathrm{m}^{-1} \mathrm{~K}^{-1}$ for $90 \mathrm{wt} \% \mathrm{FBN}$ loading in FBN/PVA film), which is nearly 100 times larger than the pristine PVA film. Meanwhile, the FBN/polymer films could provide good fire-resistance ability, thus effectively retarding the flammability. In addition, the nanocomposite film with high concentration of FBN up to $70 \mathrm{wt} \%$ still remains excellent flexibility and toughness even after 100 times rolling and folding. Interestingly, the rolled film hollow cylinder could support 25,000 times its own weight without fragile crack, highlighting the extra strong interaction between the FBN and PVA. Those properties make it an excellent candidate for thermal management in electronics.
\end{abstract}

\section{Introduction}

Traditional electron devices and electric appliances such as cell phones and televisions are becoming more intelligentized and personalized. However, although these devices have integrated the advantages of smaller size and multi-functions, the issue of low heat dissipation still remains in suspense. Especially with the frequent use of electronics, the batteries consume faster than before, thus generating intense heat and causing accessories failure. ${ }^{1}$ Therefore,

a Institute for Frontier Materials, Deakin University, Waurn Ponds Campus, Locked Bag 20000, Victoria 3220, Australia.

${ }^{b}$ School of Materials Science and Engineering, Southwest Petroleum University, Chengdu 610500, PR China.

c Department of Physics and Astronomy and Department of Biomedical Engineering, Rowan University, 201 Mullica Hill Road, Glassboro, New Jersey 08028, United States. ${ }^{d}$ International Center for Materials Nanoarchitectonics (WPI-MANA), National Institute for Materials Science (NIMS), Namiki 1-1, Tsukuba, Ibaraki 305-0044, Japan.

e College of Engineering and Applied Sciences, Nanjing University, Nanjing 210093, China.

${ }^{f}$ Australian Institute for Innovative Materials University of Wollongong North Wollongong, NSW 2500, Australia.

$\dagger$ J. Wang and Y. Wu have contributed equally.

*Corresponding authors should be addressed at: dan.liu@deakin.edu.au (D. Liu) and weiwei.lei@deakin.edu.au (W.W. Lei).

Electronic Supplementary Information (ESI) available: details of any supplementary information available should be included here. See DOI: 10.1039/x0xx00000x to design materials with efficient thermal management is of high significance. Recent studies have focused on the incorporation of nanomaterials such as carbon nanotubes (CNTs), graphene and boron nitride (BN) nanosheets into polymers to enhance the thermal conductivity. ${ }^{2-5}$ Nevertheless, it is difficult to employ CNTs and graphene as electronic packaging materials owing to their electrical conductivity and fast oxidation of carbon. On the contrary, BN nanosheets (BNNS) offer a high thermal conductivity, large and direct band gap, resistance to oxidation, high elastic modulus and a low friction coefficient, which suggest utility in thermal management applications in electronics. ${ }^{6-11}$

There have been many reports on BNNS-polymer nanocomposites for thermal conductivity improvement. ${ }^{4}, 12$ However, two main challenges still keep to be solved in fabricating the nanocomposites. One is the low fractions of BNNS in the polymer matrix. In most cases, the total thermal conductivities of composites are largely controlled by the polymers, staying far from the inherent properties of BNNS. Thus the BNNS/polymer composites normally remain low thermal conductivity $\left(1-3 \mathrm{~W} \mathrm{~m}^{-1} \mathrm{~K}^{-1}\right){ }^{4,13}$ To address this issue, some works devoted to increase the contents of BNNS. Unfortunately, despite the thermal properties improvement, the whole mechanical performance of the nanocomposites largely deteriorates due to the brittleness of BNNS. ${ }^{14-17}$ Another challenge is the poor interface miscibility of BNNS and polymers. In the nanocomposites, the 
aggregation of BNNS and poor interaction with polymers greatly impede the thermal conductivity enhancement. ${ }^{18-22}$ To solve this problem, some studies adopted some chemical agents to functionalize BNNS. ${ }^{23,24}$ However, the thermal conductivity still remains low due to the large size of the BNNS. ${ }^{4,25-27}$ Therefore, to simplify the functionalization of BNNS with highly water-soluble, as well as improving the dispersibility in polymer matrix is the key to both thermal conduction and mechanical property enhancement.

In a previous report, we have synthesized an amino functionalized BN (FBN) nanosheets, which could be highly dispersible and stable in water. ${ }^{28}$ Herein, we successfully fabricate a free-standing FBN nanosheets/polymer composite film with layer by layer laminate nanostructures by facile vacuum filtration of FBN nanosheets and water soluble polymers such as polyvinyl alcohol (PVA). It is worth noting that, except for water, no other organic solvents such as isopropanol or ethanol are used in the preparation protocol. More importantly, different from most functional BN nanosheets with limited dispersibility and low concentration in aqueous polymer matrix, our FBN nanosheets could be mixed with water soluble polymer at any weight proportions without precipitation. An ultrahigh in-plane thermal conductivity of $120 \mathrm{~W} \mathrm{~m}^{-1} \mathrm{~K}^{-1}$ for FBN/PVA 10 wt\% film is achieved, with nearly $12000 \%$ enhancement from the pristine PVA film. In addition, the film also exhibits good fire resistance, remaining the initial shape after burning for more than 60 s. Moreover, the nanocomposite film is flexible and robust enough even at a high FBN loading (70 wt\%). The ultra-high thermal conductivity and excellent mechanical property of our FBN/PVA nanocomposites attribute to the super compatibility between FBN nanosheets and polymer chains, which originates from the hydrogen bonding effect. To demonstrate the strong interaction and super compatibility, for the first time, we adopt Pickering emulsion formed by FBN and PVA in the $\mathrm{n}$-hexane and water interface. The strong hydrogen bonding interaction produces a homogenous, robust and stable FBN/PVA emulsion throughout the oil/water system, interpreting the intimate combination of FBN and PVA, which is even better than that of oxide graphene (GO)/PVA in the same condition.

\section{Experimental}

Preparation of functionalized boron nitride (FBN) nanosheets. FBN nanosheets were prepared using a previously reported method. ${ }^{28}$ Typically, h-BN (Momentive Performance Materials, Inc.) and urea (Sigma-Aldrich) were mixed in a steel milling container using a planetary ball mill (Pulverisette 7, Fritsch). The mixture with a weight ratio of 1:20 (h-BN: urea) was protected by nitrogen and milled at a rotation speed of 500 r.p.m. for 20 h. The obtained powders were dissolved in water and dialyzed for 1 week in deionized water to remove the urea, yielding stable aqueous dispersions of BN nanosheets.

Preparation of FBN/polymer films.

FBN/polymer films were fabricated by vacuum-assisted filtration (VAF). The aqueous solution of $B N$ nanosheets $(3-4 \mathrm{mg} / \mathrm{ml}$ ) was mixed with PVA water solution $(2 \mathrm{mg} / \mathrm{ml}$ ) according to the different weight ratios. Then the mixtures were ultrasonicated in a water bath for $10 \mathrm{~min}$. To form composite films, the mixture was filtered through a glass microfiltration apparatus (Sigma) of ca. $36 \mathrm{~mm}$ in diameter with a polyethylene membrane under vacuum assistance. These films can be readily peeled off from the polyethylene membranesand retain their freestanding state. The mass fractions 3 of PVA FBN/polymer mixtures were set as 10, 20, 30, 40, 50, and $60 \mathrm{wt} \%$, and the resulting films were denoted as FBN/PVA 10, 20, 30, 40, 50, and $60 \mathrm{wt} \%$, respectively. Pure FBN films were produced using a similar approach and were denoted as FBN.

\section{Material characterization.}

XRD measurements were performed on a PANalytical X'Pert PRO apparatus operating with $\mathrm{Cu} K \alpha$ radiation. SEM analysis was performed on a Zeiss Supra 55 VP SEM instrument. TEM and HRTEM imaging were performed on a JEOL $2100 \mathrm{~F}$ microscope operating at $200 \mathrm{kV}$. The FTIR and optical transmittance spectra were recorded using a Nicolet 7199 FTIR spectrometer and Cary 3 spectrophotometer, respectively. The thermal behavior was analyzed using TGA on a TA Instruments Q50 TGA thermal analyzer at a heating rate of $10{ }^{\circ} \mathrm{C} \mathrm{min}^{-1}$ from room temperature to $800{ }^{\circ} \mathrm{C}$ under $60 \mathrm{sccm}$ compressed air flow. Specific heat was measured by using the differential scanning calorimetry (DSC Q200, TA Instruments).

\section{Pickering emulsion preparation.}

Typically, pure PVA, pure FBN and FBN/PVA (10 wt\% - 90 wt\%) aqueous dispersions $(2 \mathrm{mg} / \mathrm{ml})$ are mixed with equal volume of $\mathrm{n}$ hexane in a vial. Then the emulsion would form shaking the liquid mixtures by hand for around $1 \mathrm{~min}$. For the comparison of FBN/PVA and GO/PVA emulsifiers, both FBN/PVA 50 wt\% emulsion $(2 \mathrm{mg} / \mathrm{ml}$ ) and GO/PVA 50 wt\% emulsion $(2 \mathrm{mg} / \mathrm{ml}$ ) are prepared in two vials respectively. After solidification for several hours, the two vials are put upside down for the stability observation.

\section{Mechanical testing.}

The FBN/PVA films and FBN films were cut into strips measuring 25 $\mathrm{mm} \times 5 \mathrm{~mm}$ in size and glued onto supporting paper frames. These cut films were fixed in the grips of a universal testing machine (Instron 2360), and then the paper frames were cut and the tensile tests were performed using a $50-\mathrm{N}$ load cell with a loading rate of 5 $\mathrm{mm} / \mathrm{min}$. These mechanical tests were conducted at room temperature under a humidity of approximately $45 \%$. The Young's modulus, tensile strength, and strain to failure were calculated as the averages of the results of five parallel experiments. The hollow cylinders of FBN/PVA $30 \mathrm{wt} \%$ film were produced by rolling the corresponding films $(34 \mathrm{~mm} \times 10 \mathrm{~mm})$ around a glass rod $(6 \mathrm{~mm}$ diameter) and gluing the edges of the strips with a small amount of PVA solution (0.1 wt\%). These hollow cylinder specimens were dried in a vacuum oven and weighed, and then they were fixed on a glass slide by PVA solution (1 wt\%) for evaluating the weight loading.

Thermal conductivity.

The in-plane thermal conductivity of the FBN/PVA films was measured by a Physical Property Measurement System (PPMS, Quantum Design, USA) using the steady-state method (Fig. S1). A film sample with a radiation shield was placed in a vacuum chamber $\left(9.4 \times 10^{-5} \mathrm{Torr}\right)$ to minimize the radiation, convection, and conduction heat loss. The radiation heat loss is also calculated by the measurement system. Usually, the specimen is $4 \mathrm{~mm} \times 18 \mathrm{~mm}$ with a rectangular shape. A heater shoe is connected to one end of the sample, and the other end is fixed to a cold sink. The hot thermometer shoe and cold thermometer shoe are both connected to the measured sample at a distance $L$ (as shown in Fig. S1). At 
steady state, the thermal conductivity (K) of the sample is determined by equation:

$\mathrm{K}=(\mathrm{Q} \times \mathrm{L}) /(\mathrm{A} \times \Delta \mathrm{T})$

where $Q$ is the net heat flowing through a known cross section $A$ of the measured sample, $\Delta T$ is the temperature difference between the hot thermometer and cold thermometer, and $L$ is the distance between the two thermometer shoes. The thermal conductivity (through-plane direction) of the BN/PVA films was analyzed by the device of ai-Phase Mobile $1 \mathrm{u}$ using a temperature-wave-analysis method according to ISO22007-3 (Supporting Information). The samples were sandwiched between the heater and sensor plates. By scanning the frequency of heat source, the delay in the phase of the temperature wave was recorded within the range from $-180^{\circ}$ to $230^{\circ}$ based on an empirical criterion. The linear fitting of phase lag $\theta$ versus square root of frequency, sqrt $(f)$, was conducted to calculate thermal diffusivity $a$ following:

$\theta=\mathrm{d} \times \mathrm{sqrt}(\pi \mathrm{f} / \mathrm{a})$

where $d$ is the thickness of samples. The thermal conductivity was further calculated based on the thermal diffusivity as well as theoretical density and specific heat capacity of composites. The experimental errors were estimated by means of the square-rootsum error propagation approach, including the following error sources: the measurement of thickness of samples and the physical property measurement system.

Fire retardancy testing.

A parallel flame test was performed on FBN/PVA $10 \mathrm{wt} \%$ films with dimensions of $20 \mathrm{~mm}$ in length and $5 \mathrm{~mm}$ in width. The fire from a gas burner was applied on the tested film for $60 \mathrm{~s}$ and then removed.

\section{Results and Discussion}

\subsection{The morphology and structures of the FBN/PVA films}

FBN nanosheets were prepared according to our previously reported method. ${ }^{28}$ By simply ball milling with urea, the BN nanosheets are easily covalently bonded with amino group. Notably, unlike the isopropanol or ethanol exfoliated BNNS which exhibit limited hydrophilicity, ${ }^{1,25}$ the FBN nanosheets with 200-300 nm lateral size could be well dispersed in water even at a high concentration of 30 $\mathrm{mg} / \mathrm{ml}$ (Fig. S2). ${ }^{28}$ After mixing, the FBN/PVA solutions are well prepared (Fig. 1a). In this article, the mass fractions of PVA in the $\mathrm{FBN} /$ polymer composite are set as 10, 20, 30, 40, 50, and $60 \mathrm{wt} \%$, and the resulting films are denoted as FBN/PVA 10, 20, 30, 40, 50, and $60 \mathrm{wt} \%$, respectively. Fig. $1 \mathrm{~b}$ displays the free-standing film of FBN/PVA 30 wt\% by vacuum filtering. It could be seen that the film is still optically transparent even at 70\% FBN loading. Other FBN/PVA (10 wt\%, $20 \mathrm{wt} \%, 40 \mathrm{wt} \%, 50 \mathrm{wt} \%$ and $60 \mathrm{wt} \%$ ) films are all transparent as well (Fig. S3). Besides, the transmittance of the film starts from $23 \%$ at $400 \mathrm{~nm}$, increases with the wavelength and reaches $73 \%$ at $800 \mathrm{~nm}$ (Fig. S4). The pronounced optical transmittance indicates the uniform mixing and homogenous distribution of FBN nanosheets and PVA. Generally, the pure BNNS membrane is too fragile to be folded. However, our FBN/PVA 30 wt\% film can be easily rolled around a glass rod or folded into an airplane shape (Fig. 1c and d), demonstrating its excellent flexibility. Importantly, the rolled film is able to self-recover its original shape without any visible damage (Fig. 1e), highlighting the effect of strong hydrogen bonding interaction that attracts thel: $10.1039 / \mathrm{CTTCO4860B}$
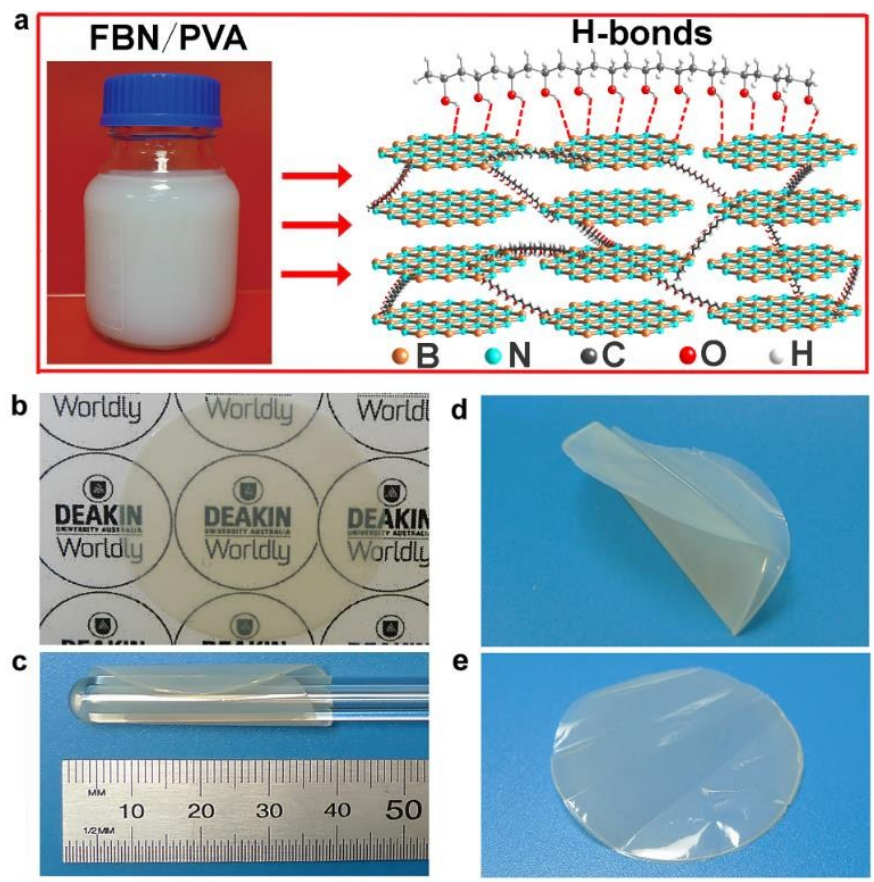

Fig. 1 (a) Photo and schematic illustration of the homogenous mixing of the FBN nanosheets and PVA solutions. (b) Photo of a free-standing FBN/PVA 30 wt\% film. (c) Photo of a piece of a FBN/PVA 30 wt\% film that was rolled around a glass rod. (d) Folded into a paper airplane shape. (e) The folded film recovered to its original shape without observable damage.

PVA chains to weave the FBN nanosheets, thereby greatly improving the extensibility and plasticity (Fig. 1e). Even after more than 100 times of "rolling-recovering" cycles, the film still remains its pristine shape (Movie S1), which is robust enough and compared to the pure PVA membrane. The internal structure and morphology of the films are investigated by scanning electron microscopy (SEM) and transmission electron microscopy (TEM). As shown in Fig. 2a and b, the pure PVA film are homogenously distributed, whereas the FBN/polymer films are composed of parallel layer by layer laminate. Previous study has pointed that an ordered "brick-and-mortar" stacked model could be formed by vacuum filtering functionalized BNNS and PVA. ${ }^{14}$ However, the stacked structure seems loosely piling up with thick layers (micron scale). In our case, the thin FBN nanosheets and PVA are closely compacted (nanoscale) (Fig. 2c), which, in turn, reveals the strong hydrogen bonding interaction, adhering the FBN nanosheets by PVA chains and constructing the extra thin heterojunction-like layer by layer laminate. Thereby, from morphology analysis, it is believed that the strong interaction between FBN nanosheets and PVA is affordable to result in the super compatibility. This is further demonstrated through a Pickering emulsion. From Fig. $2 d$, it could be seen that, after shaking the $n$ hexane/water systems, pure PVA could form a suspension emulsion because of the hydrophilicity of hydroxyl group and hydrophobicity of alkyl in the polymer chains. Whereas pure FBN nanosheets which is highly hydrophilic are not wettable in $n$-hexane, leading to a hierarchy of oil and water in the system. Here $n$-hexane is employed 
as oil phase rather than toluene when considering the elimination of $\pi-\pi$ conjugation effect between FBN and organic agents with benzene ring. Interestingly, after mixing FBN with PVA, the well
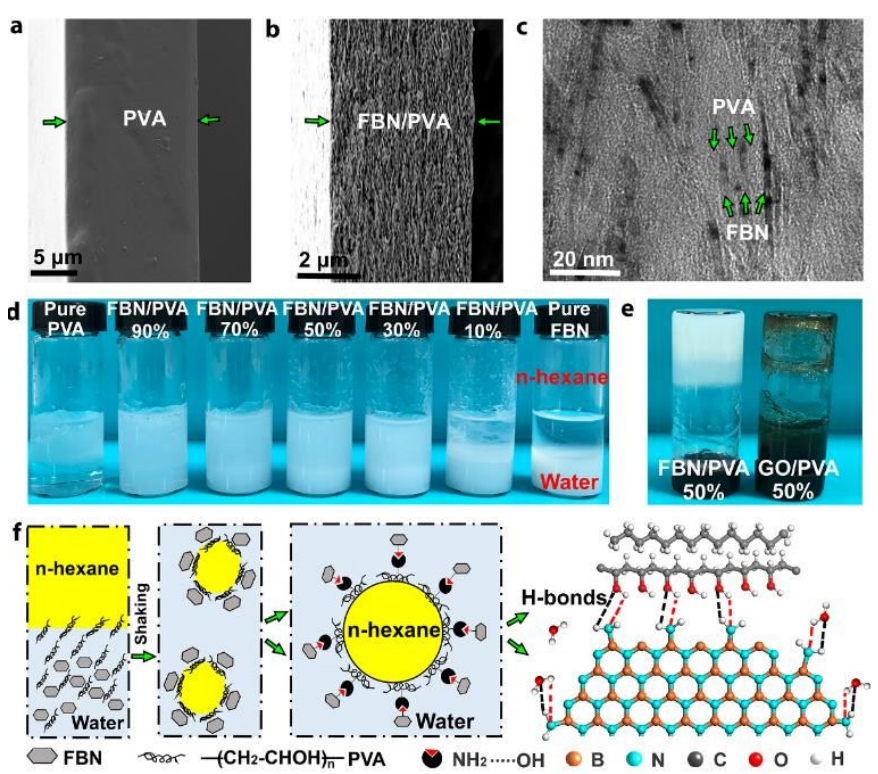

Fig. 2 (a) Cross-sectional SEM images of pure PVA and (b) FBN/PVA $30 \%$ film. (c) Cross-sectional TEM image of FBN/PVA 30 wt $\%$ film. (d) Pickering emulsion of FBN/PVA ( $2 \mathrm{mg} / \mathrm{ml}$ ) with different contents in nhexane/water, the emulsion is formed by hand shaken for 1 minute. (e) The solidification and stabilization of emulsifier by FBN/PVA 50 wt\% and GO/PVA $50 \mathrm{wt} \%$ (2 $\mathrm{mg} / \mathrm{ml})$. (f) The possible mechanism scheme of the formation of Pickering emulsion derived from FBN nanosheets and PVA.

dispersed Pickering emulsions are achieved. It is known that the Picker emulsion is stabilized by solid particles rather than molecular surfactants. ${ }^{29}$ Although PVA acts as an amphiphile here, however, the whole stable emulsion rather than suspension develops owing to the strong hydrogen bonding interaction between FBN nanosheets and PVA chains. When $10 \mathrm{wt} \%$ of PVA is added, some floccule begins to appear in the $n$-hexane, suggesting an obvious oil-in-water $(\mathrm{o} / \mathrm{w})$ process. ${ }^{30}$ After $30 \mathrm{wt} \%$ or higher PVA contents added, the well dispersed latescence is generated without any coalescence. Obviously, the hydrogen bonds attraction magnifies with the PVA contents increasing and functions as a binding force here, surpassing the gravity and fluid resistance, driving the FBN nanosheets into the oil/water interface and finally constructing the milky emulsion. X-ray diffraction (XRD) results (Fig. S5) of FBN/PVA composites also reflect this trend. With the increasing incorporation of PVA, especially higher than $30 \mathrm{wt} \%$, the (002) peak of FBN shifts to a higher diffraction angle with remarkably reduced intensity, indicating that the spacing between the layers of FBN slightly decreases with the increasing amount of PVA. This is also consistent with the TEM results and certifies that the polymers chains can enter the space between FBN nanosheets. Fourier-transform infrared spectroscopy (FTIR) (Fig. S6) further discloses that the characteristic peaks of FBN decrease in intensity and shift with increment of PVA, which is in agreement with the results obtained from graphene oxide $(\mathrm{GO})$ combined with PVA, ascribing to the strong $\mathrm{H}$-bonding interaction between the $\mathrm{FBN}$ nanosheets and PVA. ${ }^{31}$ More importantly, the FBN/PVA emulsion is highly stable as shown in Fig. 2e. When placed in ithe $_{\text {r }}$ ambiemt atmosphere for several hours, the solidified 1 PBNYPNAT $50486 \% \%$ emulsion system is robust enough to suspend in the air against gravity. While the GO/PVA emulsion with the same concentration could not remain that stable and immediately drops down after hanging. It could be speculated that, after emulsion, the amphiphilic moieties originate from the alkane of PVA chain and amino group of FBN nanosheets whereas the hydroxyl group of PVA only bridges the FBN nanosheets through hydrogen bonding with amino group (Fig. 2f). This is why pure PVA or FBN nanosheets could not construct a whole Pickering emulsion independently while the mixture of FBN/PVA could form it. All the results highlight the super compatibility and intimate incorporation of FBN nanosheets and PVA polymer chains, which further improve the mechanical and thermal properties of the composite to a large extent.

\subsection{The Mechanical and thermal properties of the FBN/PVA films}

Unlike other 2D films, including GO or graphene films, ${ }^{5}$ which exhibit good mechanical properties, pure FBN nanosheet films are brittle. The Young's modulus and tensile strength of the FBN films are 28.3 \pm 10.6 and $7 \pm 0.4 \mathrm{MPa}$, respectively (Table S1). These values are rather low compared with those of the $2 \mathrm{D}$ films mentioned above and far below that of single-crystal h-BN ( $36.5 \mathrm{GPa})$ due to weak bonding between the multi-layer BN sheets in the films. ${ }^{32}$ However, upon introducing PVA, the Young's modulus and tensile strength are greatly improved. As shown in Fig. 3a, b and Table S1, upon introducing 10 wt\% PVA into the FBN nanosheet films, the Young's modulus and tensile strength are improved by approximately 50 and 30 times, respectively. The strain to failure is also enhanced by the addition of more PVA (Fig. S7). The mechanical properties of other FBN/polymer films with different polymer contents are shown in Fig. $3 a$ and $b$, indicating that the mechanical properties of the composite is tailorable by changing the weight ratios of FBN nanosheets to PVA. Notably, the tensile strength dramatically increases from FBN/PVA $30 \mathrm{wt} \%$ and peaks at FBN/PVA $40 \mathrm{wt} \%$, which is in a good accordance with the phenomenon in the Pickering emulsion that FBN/PVA 30 $w t \%$ starts to act as robust emulsifier due to the strong enough hydrogen bonding interaction.

To observe the compressive strengths of the composite films, hollow cylinder made by rolling and pasting the overlapping edges of FBN/PVA films is used in weight-supporting applications. As shown in Fig. 3a inset, a hollow cylinder of FBN/PVA $30 \mathrm{wt} \%$ (6 mm in diameter and $10 \mathrm{~mm}$ high), made from a film of $34 \mathrm{~mm}$ in length and $10 \mathrm{~mm}$ in width can readily support approximately 25,000 times its own weight without fragile crack, which is significantly higher than those of other 2D nanosheet/polymer films. ${ }^{33}$ The robust compressive strength and toughness of the composite films further demonstrate the strong interfacial adhesion and super compatibility between FBN nanosheets and PVA. The high compressive strength and toughness, together with the exceptional flexibility make these FBN/PVA films promising as packaging materials in flexible electronic devices and temperature sensors. The FBN/PVA films exhibit not only the excellent mechanical properties but also highly anisotropic thermal conductivity. The thermal conductivities of FBN/PVA films with 

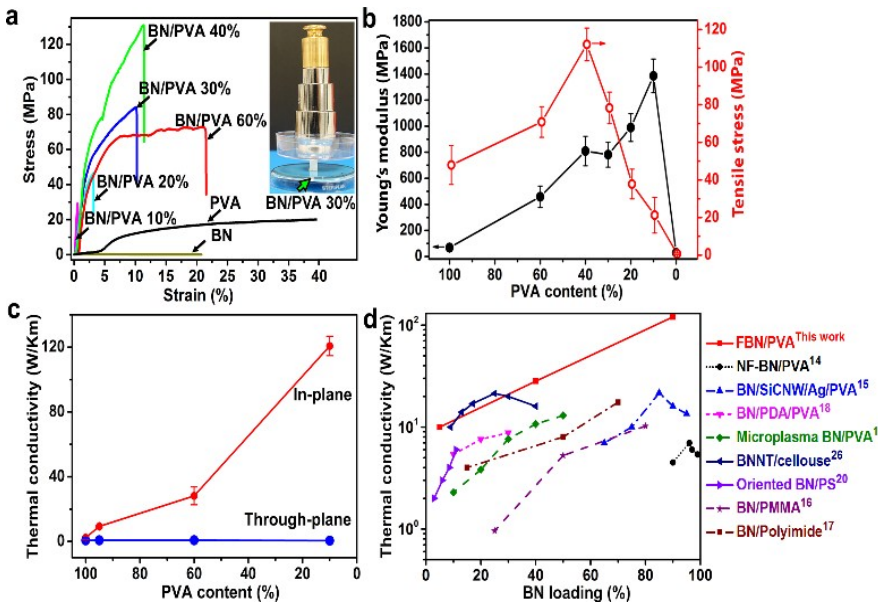

Fig. 3 Mechanical and thermal properties of FBN, FBN/PVA, and PVA films. (a) Stress-strain curves of FBN, FBN/PVA, and PVA films; inset photo shows a FBN/PVA 30 wt\% film supporting 25,000 times its own weight. (b) Young's modulus and tensile stress with different PVA contents. (c) In-plane and through-plane thermal conductivities of FBN/PVA films with different contents of polymer. (d) Thermal conductivity comparison of the FBN/PVA and other BN/polymer composites reported in previous work.

different polymer weight percentages are presented in Fig. 3c. The in-plane thermal conductivity can be high up to $120.7 \mathrm{~W} \mathrm{~m}^{-1} \mathrm{~K}^{-1}$ for FBN/PVA 10 wt\%, nearly 100 times larger than the pristine PVA film. Even for FBN/PVA $60 \mathrm{wt} \%$ film, the in-plane thermal conductivity value can reach $28.2 \mathrm{~W} \mathrm{~m}^{-1} \mathrm{~K}^{-1}, 3000 \%$ enhancement at such relative low BN loading. In order to demonstrate the superiority thermal conductivity of the FBN/PVA film, Fig. 3d summarizes previously reported thermal conductivity enhancement of $\mathrm{FBN} /$ polymer composites with different $\mathrm{FBN}$ weight contents in the polymer matrix. ${ }^{14-20,26}$ It can be seen that the FBN/PVA film exhibits the highest thermal conductivity enhancement with the similar BN weight content range among the reported BN/PVA or other polymer composites, which attributes to not only the high thermal conductivity of FBN nanosheets, but also the strong $\mathrm{H}$-bonding interaction and high miscibility. In fact, it has been found that interpolymer $\mathrm{H}$-bonding-capable moieties and linker units could essentially yield a dense and homogeneously distributed thermal network, benefiting the increment of in cross-plane thermal conductance. ${ }^{34}$ Therefore, the establishment of effective thermal network between FBN nanosheets and PVA via strong $\mathrm{H}$-bonding leads to the extra high in-plane thermal conductivity. Moreover, it is notable that the FBN/polymer films also display a large anisotropy of thermal conductivity with an extremely low through-plane thermal conductivity $\left(1.1 \mathrm{~W} \mathrm{~m}^{-1} \mathrm{~K}^{-1}\right)$. The anisotropy of the thermal conductivity of the composite film originates from the strong anisotropic thermal conductivity of BN nanosheets. ${ }^{12-14}$ Furthermore, the layered composite film consisting of the FBN nanosheets aligned in the polymers due to self-assembly of FBN nanosheets sandwiched by very thin layers of polymer during vacuum filtration which further facilitates the heat transfer along the in-plane direction. Therefore, there is a large contact area between the adjacent FBN nanosheets (Fig. 2c) and the heat is preferably transferred along the FBN nanosheets in-plane direction. With more FBN nanosheets aligned in-plane direction, the interfacial thermal resistance decreases and the number of conductive paths for in-plane heat transfer $r_{A}$ increeasese

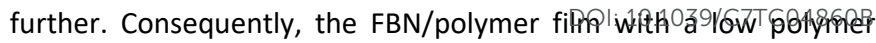
content exhibits a relatively high in-plane thermal conductivity. On the contrary, polymer presented between the FBN nanosheets in through-plane direction leads to slow heat transfer and large anisotropy, impeding the heat transfer in the through-plane direction. ${ }^{26}$ Hence the FBN/PVA films show a great in-plane thermal conductivity and a lower thermal conductivity along the throughplane, resulting in the highly anisotropic thermal conductivity.
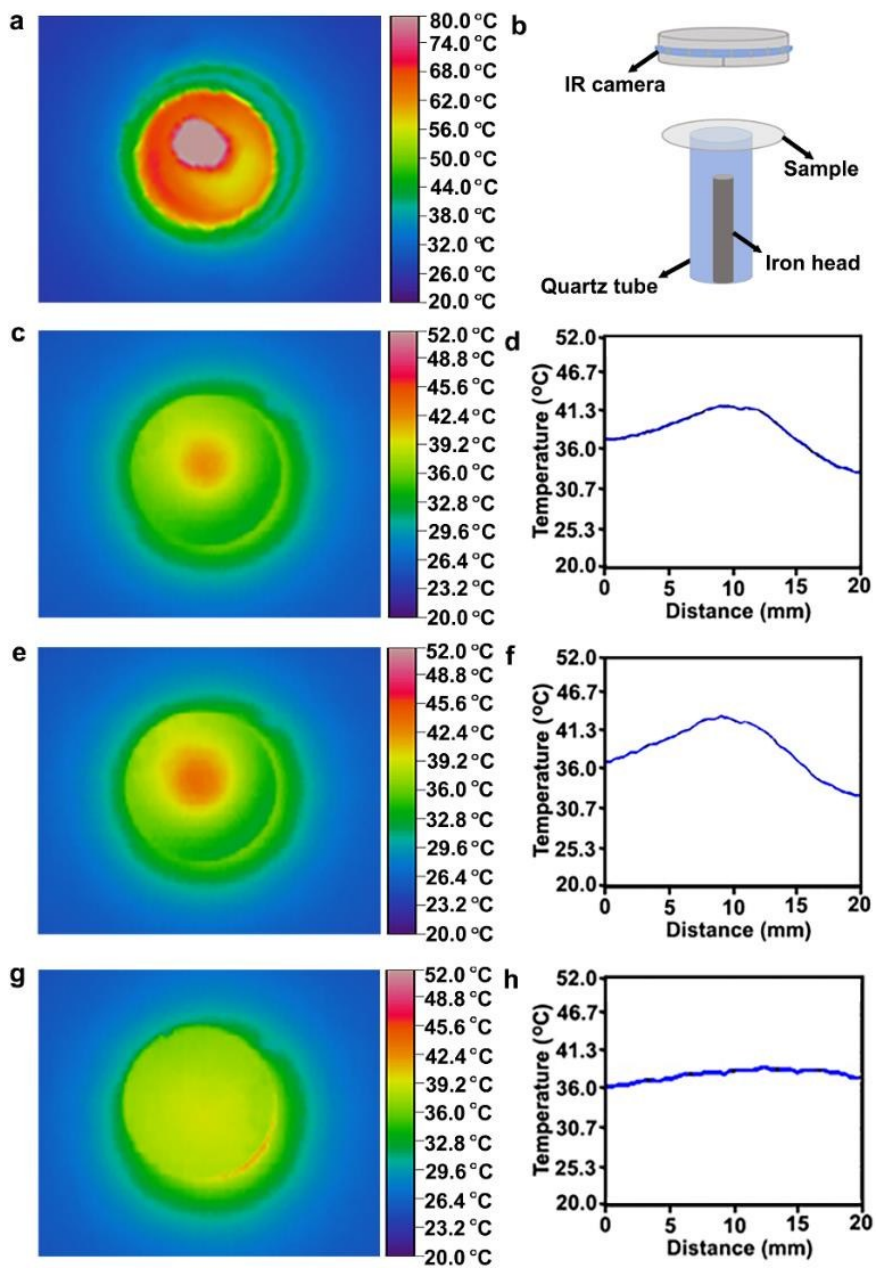

Fig. 4 Anisotropic thermal conductivity of FBN/PVA films. (a) Photograph of an iron head playing the role of a hot spot recorded by an infrared thermal camera and (b) experimental configuration. Steadystate temperature distributions of samples suspended above the iron head (c) $\mathrm{Cu},(\mathrm{e}) \mathrm{Al}$, and (g) FBN/PVA $10 \mathrm{wt} \%$ film with the same thickness of $20 \mathrm{~mm}$ and their temperature profiles $(\mathrm{d}, \mathrm{f}, \mathrm{h})$ at the centerline of the sample.

\subsection{The high thermal conductivity anisotropy of the FBN/PVA films}

The highly anisotropic thermal conductivity behavior of our FBN/PVA films is further demonstrated by comparison with $\mathrm{Cu}$ and Al foils in a heat transfer experiment (Fig. 4). The tested samples are put on a hot iron head, and an infrared thermal camera is used to record the temperature distribution under a steady-state (Fig. 4b). To ensure consistent emissivity on the surfaces of metals and FBN/PVA films as 
well as the thermal radiation transfer between the hot iron head and the samples, a thin layer of graphite is spray-coated on both top and bottom surfaces. Fig. 4a shows that the heat from the iron head is concentrated. When the iron head is covered by $\mathrm{Cu}$ or Al foil, a hot spot appears at the center with a high temperature gradient from
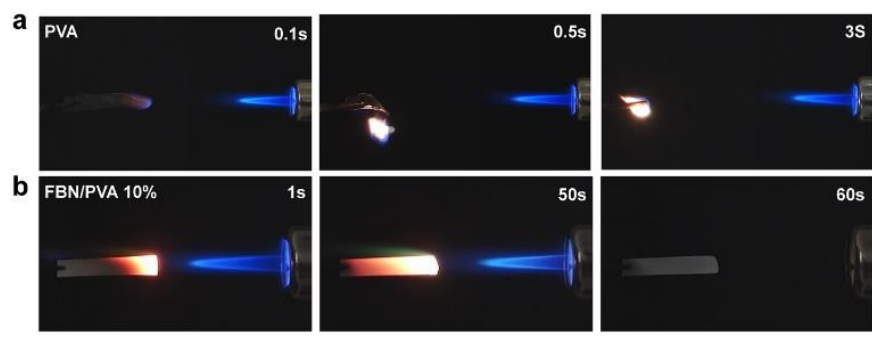

Fig. 5 Fire-retardant properties of FBN/PVA films. Photographs of (a) pure PVA and (b) FBN/PVA 10\% films on a hot flame with different times.

the center to the edge of the metal foil (Fig. 4c-f). However, the FBN/PVA 10 wt\% film exhibits a dramatically reduced center spot temperature with a more uniform temperature distribution under similar conditions (Fig. 4g and h). The FBN/polymer films exhibit highly anisotropic properties, well corresponding to the thermal conductivity results (Fig. $3 \mathrm{c}$ ). It indicates that the heat is not conducted in the out-of-plane direction, but being spread in-plane direction efficiently. Consequently, the concentrated hot spot could not be clearly presented on the FBN/PVA film in Fig. 4g. Therefore, the FBN/PVA films fabricated here are promising thermally conductive composite materials as well as providing excellent electrical insulation, which find intriguing applications in thermal management (heat spreading), flexible thermal and electrical insulation, and electrically insulating substrates in electronics.

\subsection{The fire retardant ability of the FBN/PVA films}

Furthermore, $\mathrm{h}-\mathrm{BN}$ is available for the fire retardant coating for wood substrates. ${ }^{35}$ Herein the FBN/PVA films could also serve as an ideal candidate in the construction of fire retardant materials. Parallel burning tests (Fig. 5a, b, and Movie S2) show that FBN/PVA 10 wt\% displays excellent fire retardant. The tested film can nearly maintain their initial shape after burning for more than $60 \mathrm{~s}$, due to the super compatibility and homogenous distribution of FBN nanosheets in polymer matrix, enabling the preservation of the flame retardant functions of films. Even when the PVA contents increase to $30 \mathrm{wt} \%$, the composite film still remains intact after ignition, demonstrating the good fire retardancy (Fig. S8a). However, the pure PVA film ignited on the flame is burnt out within only 4s (Fig. 5a). Thermogravimetric analysis (TGA) in air reveals that the oxidation resistance of the FBN/PVA films increases with the FBN content (Fig. $\mathrm{S} 8 \mathrm{~b})$. The superior flame retardant property of the FBN/polymer films may significantly improve the working safety when employed as heat shields, flexible fire retardant coatings, and electrically insulating substrates.

\section{Conclusions}

In summary, nearly transparent, flexible and tough ${ }_{A}$ FBN/PVA

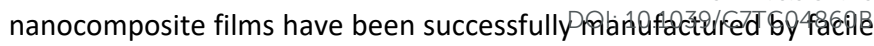
but scalable vacuum assisted filtration. The as-obtained films have demonstrated both impressive fire-retardant ability and superior inplane thermal conductivity: $28.2-120.7 \mathrm{~W} \mathrm{~m}^{-1} \mathrm{~K}^{-1}$ at $40-90 \mathrm{wt} \%$ of FBN loading, enhancement of $3000 \%-12000 \%$ based on the pure PVA. The pronounced thermal conductive performance could be ascribed to the extra strong $\mathrm{H}$-bonding interaction and super compatibility between FBN nanosheets and PVA chains, which is also manifested by the robust FBN/PVA Pickering emulsifier. In addition, the composite films also display excellent rolling-recover flexibility and compressive toughness even at high concentration of FBN (70 wt\%). Those properties, combined with high chemical and temperature stability make the FBN/polymer films useful for various applications, particularly in thermal management (heat spreading), flexible and fire-retardant electronic devices.

\section{Acknowledgements}

This work was financially supported by the Australian Research Council Discovery Early Career Researcher Award scheme (DE150101617 and DE140100716), and Deakin University, Central Research Grant Scheme. J. Wang and Y. Wu have contributed equally.

\section{Notes and references}

1 H. Zhu, Y. Li, Z. Fang, J. Xu, F. Cao, J. Wan, C. Preston, B.Yang, L.B. Hu, ACS nano 2014, 8, 3606-3613.

2 Z. Han, A. Fina, Prog. Polym. Sci. 2011, 36, 914-944.

3 T. Ramanathan, A. A. Abdala, S. Stankovich, D. A. Dikin, M. Herrera-Alonso, R. D. Piner, D. H. Adamson, H. C. Schniepp, X. Chen, R. S. Ruoff, S. T. Nguyen, I. A. Aksay, R. K. Prud'Homme, L. C. Brinson, Nature nanotech. 2008, 3, 327-331.

4 W. Meng, Y. Huang, Y. Fu, Z. Wang, C. Zhi, J. Mater. Chem. C 2014, 2, 10049-10061.

5 L. Peng, Z. Xu, Z. Liu, Y. Guo, P. Li, C. Gao, Adv. mater. 2017,29 1700589.

6 D. Liu, W. Lei, S. Qin, K. D. Klika, Y. Chen, Phys. Chem. Chem. Phys. 2016, 18, 84.

7 W. Lei, D. Liu, Y. Chen, Adv. Mater. Interfaces 2015, 2, 2.

8 W. Lei, H. Zhang, Y. Wu, B. Zhang, D. Liu, S. Qin, Z. Liu, L. Liu, Y. Ma, Y. Chen, Nano Energy 2014, 6, 219.

9 D. Liu, W. Lei, S. Qin, Y. Chen, Sci. Rep. 2014, 4, 4453.

10 D. Liu, L. He, W. Lei, K. D. Klika, L. Kong, Y. Chen, Adv. Mater. Interfaces 2015, 2, 1.

11 W. Lei, S. Qin, D. Liu, D. Portehault, Z. Liu, Y. Chen, Chem. commun. 2013, 49, 1772013.

12 W. L. Song, P. Wang, L. Cao, A. Anderson, M. J. Meziani, A. J. Farr, Y. P. Sun, Angew. Chem., Int. Ed. 2012, 51, 6498-6501.

13 J. Chen, X. Huang, Y. Zhu, P. Jiang, Adv. Funct. Mater. 2017, 27, 1604754.

14 X. Zeng, L. Ye, S. Yu, H. Li, R. Sun, J. Xu, C. Wong, Nanoscale 2015, 7, 6774-6781.

15 Y. Yao, X. Zeng, R. Sun, J. B. Xu, C. P. Wong, ACS Appl. Mater. Interfaces 2016, 8, 15645-15653.

16 T. Morishita, H. Okamoto, ACS Appl. Mater. Interfaces 2016, 8, 27064-27073.

17 M. Tanimoto, T. Yamagata, K. Miyata, S. Ando, ACS Appl. Mater. Interfaces 2013, 5, 4374-4382.

18 H. Shen, J. Guo, H. Wang, N. Zhao, J. Xu, ACS Appl. Mater. Interfaces 2015, 7, 5701-5708. 
19 R. C. Zhang, D. Sun, A. Lu, S. Askari, M. Macias-Montero, P. Joseph, D. Dixon, K. Ostrikov, P. Maguire, D. Mariotti, ACS Appl. Mater. Interfaces 2016, 8, 13567-13572.

20 X. Wang, P. Wu, ACS Appl. Mater. Interfaces 2017, 9, 1993419944.

21 F. Wang, X. Zeng, Y. Yao, R. Sun, J. Xu, C. P. Wong, Sci Rep 2016, 6, 19394.

22 F. Yuan, W. Jiao, F. Yang, W. Liu, J. Liu, Z. Xu, R. Wang, J. Mater. Chem. C 2017, 5, 6359.

23 W. Jin, L. Yuan, G. Liang, A. Gu, ACS Appl. Mater. Interfaces 2014, 6, 14931-14944.

24 J. Yu, H. Mo, P. Jiang, Polym. Advan. Technol. 2015, 26, 514520.

25 F. Xiao, S. Naficy, G. Casillas, H. Khan, T. Katkus, L. Jiang, H. Liu, H. Li, Z. Huang, Adv. mater. 2015, 27, 7196-7203.

26 X. Zeng, J. Sun, Y. Yao, R. Sun, J. B. Xu, C. P. Wong, ACS Nano 2017, 11, 5167-5178.

27 L.Jing, H. Li, R. Y. Tay, B. Sun, S. H. Tsang, O. Cometto, J. Lin, E. H. T. Teo, A. I. Y. Tok, ACS Nano 2017, 11, 3742-3751.

28 W. Lei, V. N. Mochalin, D. Liu, S. Qin, Y. Gogotsi, Y. Chen, Nat. Commun. 2015, 6, 8849.

29 J. Xu, M. Antonietti, J. Am. Chem. Soc. 2017, 139, 6026-6029. 30 J. Tang, M. F. Lee, W. Zhang, B. Zhao, R. M. Berry, K. C. Tam, Biomacromolecules 2014, 15, 3052-3060.

31 C. Bao, Y. Guo, L. Song, Y. Hu, J. Mater. Chem. 2011, 21, 1394213950.

32 U. Khan, P. May, A. O. Neill, A. P. Bell, E. Boussac, A. Martin, J. N. Coleman, Nanoscale 2013, 5, 581-587.

33 Z. Kuang, Y. Chen, Y. Lu, L. Liu, S. Hu, S. Wen, Y. Mao, L. Zhang, Small 2015, 11, 1655-1659.

34 G. H. Kim, D. Lee, A. Shanker, L. Shao, M. S. Kwon, D. Gidley, J. Kim, K. P. Pipe, Nature Mater. 2015, 14, 295-300.

35 J. Liu, R. G. Kutty, Q. Zheng, V. Eswariah, S. Sreejith, Z. Liu, Small 2017, 13, 1602456. 